\title{
Explaining the barriers and facilitators of ethnic and traditional food choices from the viewpoints of women
}

\author{
Arezoo Haghighian Roudsari ${ }^{{ }^{*}}$ (D), Abouali Vedadhir ${ }^{2}$, Jamal Rahmani ${ }^{3}$ and Ali Milani Bonab ${ }^{3}$
}

\begin{abstract}
Background and objectives: Women's food choices and preferences are important as key decision-makers in household food handling, especially the selection of local and traditional foods that can potentially affect health and disease profile. Therefore, this study aimed to understand the barriers and facilitators of choosing local and traditional foods from the perspective of women living in Iran.

Methods: This qualitative study was carried out using a semi-structured in-depth interview with 22 women aged 30-64 years old in Tehran (capital of Iran). The participants were recruited through purposive sampling with the maximum variation such as occupation, the district of residence, and education. Data collection and analysis were done simultaneously from the beginning of the study using the MAXQDA 10 software.

Results and conclusions: The four main themes explored from the participants' statements included: "cultural contexts," "social barriers and motivations," "convenience, accessibility, and skills," and "religious considerations." The lessons derived from family, experiences, beliefs, ethnicity patterns, influences from traditional medicine, and priorities in a lifetime, family preferences, routine complications, facilities and ingredients, cookery skills, and religious rules and occasions were extracted as sub-themes including the barriers and facilitators of local and traditional foods in women. Considering the diversity of local and traditional foods in Iranian culture, encouraging the varied consumption of these foods could contribute to the sustainability of diet in communities. Therefore, emphasizing the special role of Iranian women in the household, it is necessary to educate women in order to regenerate the local and traditional food patterns in our country.
\end{abstract}

Keywords: Traditional foods, Ethnic foods, Women groups, Iran, Qualitative research

\section{Introduction}

The modern view of food is not solely for satiety, but also to meet the mental needs of individuals. Food is not just a physiological need, but as a social necessity, and a symbol of subjective belongings, customs and traditions, social status, and relationships that exist in communities. Food habits are part of the societies' culture, with foodrelated cultural traditions largely based on unrefined foods, and consumers of this type of food are

\footnotetext{
*Correspondence: ahaghighian@yahoo.com

'Department of Community Nutrition, Department of Food and Nutrition Policy and Planning Research, National Nutrition and Food Technology Research Institute, Faculty of Nutrition Sciences and Food Technology, Shahid Beheshti University of Medical Science, No. 7, Hafezi (West Arghavan) St., Farahzadi Blvd., Qods Town, 1981619573P.O. Box: 19395-4741, Tehran, Iran

Full list of author information is available at the end of the article
}

theoretically more likely healthy than those who use more refined foods. For instance, infertility, heart disease, diabetes, autoimmune diseases, mental illness, obesity, dental caries, and other common diseases are more prevalent nowadays compared to past traditional societies [1].

Traditional and local foods are part of the cultural cuisine which have a discrete cultural identity and were common in societies before the modernization and industrialization [2]. Traditional and local foods, by definition, include all foods that have been prepared using local natural resources and have cultural acceptance. This term also comprises social and cultural meanings, accessibility, the ways for preparing and consuming, ingredients, and their nutritional implications [3]. From the point of view of consumers, traditional foods are

(c) The Author(s). 2019 Open Access This article is distributed under the terms of the Creative Commons Attribution 4.0 International License (http://creativecommons.org/licenses/by/4.0/), which permits unrestricted use, distribution, and 
defined as products that are frequently consumed or associated with celebrations and special occasions such as festivals, often contain local food items specific to a region or country and, in some seasons of the year, naturally transferred from a generation to another [4]. Iranian traditional foods have differences in various sub-cultures and geographic locations in terms of the antiquity, diversity, ingredients, and even the method of preparation that make them unique to the same region. Today, it has been proven that using the traditional methods such as soaking, drying, fermenting, peeling, and germination of plant foods can help improve the quality and safety of traditional foods [5].

In recent years, people have been paying more attention to this type of food, thereby expanding its consumption in the diet. People's motivations to choose traditional foods have been increased for several reasons: their effect on weight control, the price of traditional and local food, ethical considerations, convenience, natural ingredients, perceived health effects, sensory appeals, and familiarity with this type of food [6].

On the other hand, nutrition transition, as a global event, has caused changes in the quality and quantity of food consumption patterns in many countries, races, social classes, and cultures, even in different parts of a country such as Iran [7]. Iranian people have a specific food consumption pattern in their traditional diet, including mixed pilafs, variety of kebabs, khoresht (Iranian stews), dizi (abgoosht), and a variety of A'ash (Iranian thick soups) (Fig. 1) which many of these food products such as khoreshts are eaten with rice [8].

Studies show that most immigrants adopt the food culture of the destination city but do not completely lose their culture. The concentration of political, cultural, economic, and commercial centers in Tehran, as the capital of Iran, has made immigrants from across the country migrate to this city. Therefore, it is important to be aware of people's mental perceptions of traditional and local foods that are lost over time. Hence, this study
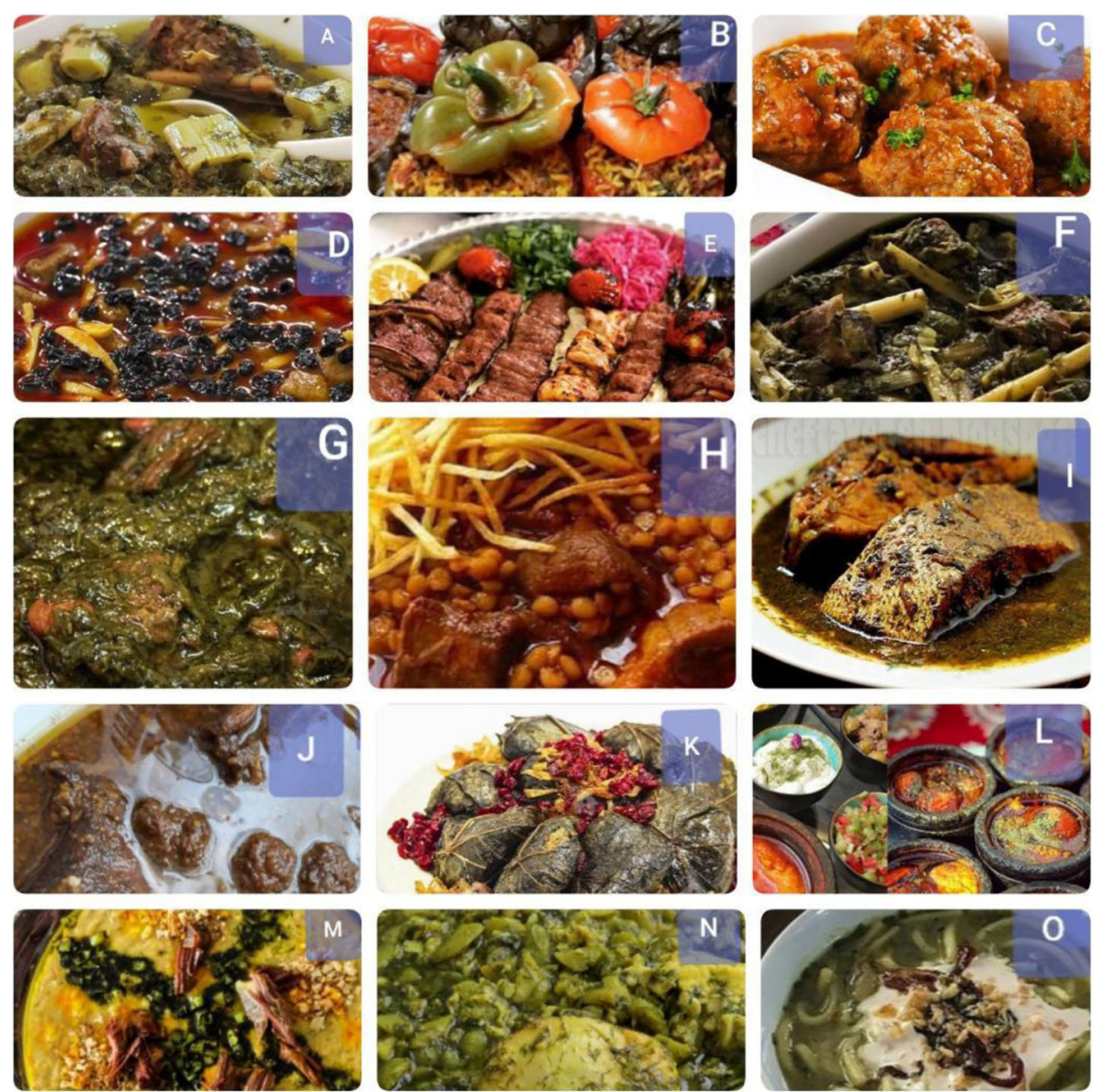

Fig. 1 Some types of traditional foods in Iran. a Rhubarb stew. b Stuffed eggplant/capsicum. c Kofta. d Almond and barbery stew. e Kebab. f Artichoke stew. $\mathbf{g}$ Herb stew/Ghormeh Sabzi. h Gheimeh. i Fish Ghalieh. j Fesenjan. k Stuffed grape leaves. I Dizi. m Bozghormeh. $\mathbf{n}$ White kidney bean stew. o Noodle thick soup/A'ash 
was conducted to explore the barriers and facilitators of choosing local and traditional foods from the perspective of women living in Tehran, due to women's food choices are important as key decision-makers in household food preparation, especially the selection of local and traditional foods that can potentially affect health and disease and achieve food security and sustainable nutrition goals.

\section{Materials and methods Methodological approach}

A qualitative design was conducted with a phenomenological approach to collect lived-experiences of women living in Tehran regarding the barriers and facilitators of choosing traditional and local foods. Using the qualitative approach allows us to gather rich data in field of the matter [9].

\section{Setting}

Tehran is the largest city and center of Tehran province located in central Iran (Fig. 2). Tehran, as the capital of Iran, is one of the major cities with a large population of residents living in other parts of the country who have migrated to this city. The metropolis of Tehran has 22 regions with many socio-economic and cultural differences in its different regions. In this study, participants were selected from different regions of Tehran.

\section{Participants}

The study population was women aged 30-64 years old who lived in Tehran. Participants were recruited using purposive sampling with a maximum variety in terms of occupation, living area, and education. They were selected from different geographical areas of Tehran that correspond to health centers, parks, cultural centers, or certain workplaces for interviewing employed women. Sampling was continued until data saturation was achieved and no new data was emerging. Interviews with 22 women resulted in data saturation and sampling was completed.

\section{Data gathering, management, and analysis}

Data collection was done using in-depth interviews including open and semi-structured questions with 22

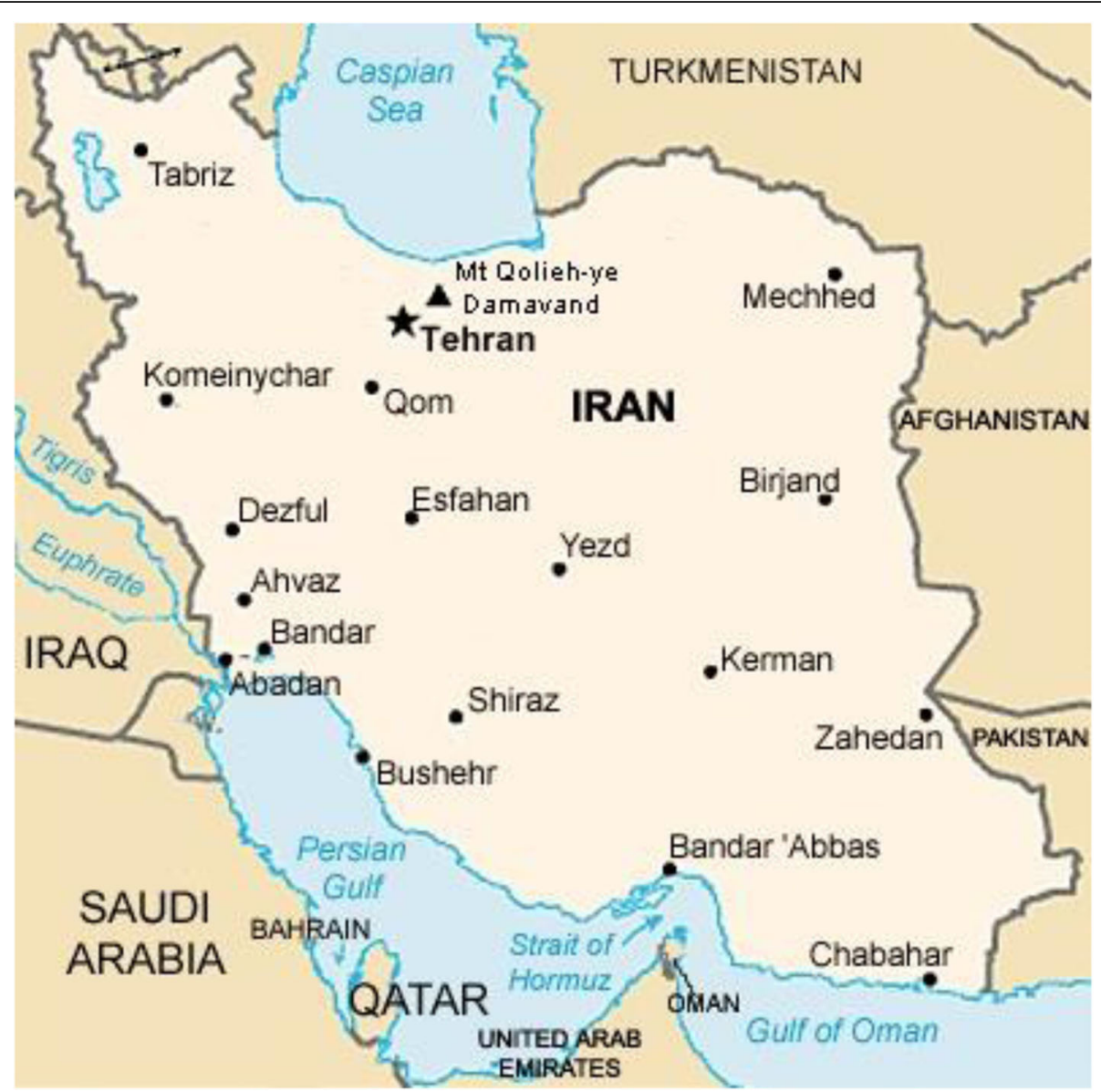

Fig. 2 Tehran as the capital of Iran among other provinces of Iran 
women aged 30-64 years old in Tehran. Data analysis was conducted using constant comparative analysis simultaneously with interviews from the beginning of the study. The interviews were recorded with permission from the interviewees, and then transcripts were imported into MAXQDA 10 software. Open coding was started and the closest concept to the participants' quotation was written in the form of a code. The coding in this study was done according to the objectives of the study, using the Strauss and Corbin method that considered the coding in three stages, open, axial, and selective [10].

\section{Data validity and trustworthiness}

To ensure the data credibility, the researcher ensured sufficient time was allocated to conduct interviews, with frequent review of the evolving data and adherence to prolonged involvement. In this study, data credibility was guaranteed by the enrollment of participants with the maximum variety and also by using several methods for gathering data, including interviewing and writing reminders. Also, with the goal of achieving confirmability, the researcher tried to avoid possible effects of prior assumptions in data collection and analysis. The data transferability was performed by providing a complete description of the characteristics of participants, the method of collection, and analysis of the data, along with the provision of some quotations of the participants, to be easily interpreted by other researchers [11].

Data reliability was also assessed through review of the data by all research team members, recording and describing all details of the research and transcribing interviews at the earliest possible time. Also, inter-coder agreement was applied using the Holsti agreement coefficient to assess the agreement between the main coder in the research team and an external coder [12].

\section{Ethical statement}

This study was supported by the research council of National Nutrition and Food Technology Research Institute and approved by the ethics committee (ethics number: IR.SBMU.nnftri.Rec.1393.053556).

\section{Results}

Women who participated in this study were aged between 30 and 64 years of age, 10 of them were employed and 12 women were housewives. Most of the participants were married and their educational level ranged from elementary to university education. After conceptualizing the codes derived from the statements of the participants, four main categories emerged relating to barriers and facilitators of choosing traditional and local foods for women living in Tehran. These include "cultural contexts," "social motivations," "convenience, accessibility, and skills," and "religious considerations." Sub-categories also emerged in each of the main categories as presented in Table 1.

According to the participants' statements, personal taste is gradually garnered over the course of life and is generally in accordance with the familial taste, modeling of parents and other cultural teachings. After childhood, food habits progressively establish based on the values of the family and society, with the mother taking the central role in family food choice which determines dietary habits in adulthood.

In addition, food choices are sometimes affected by the lack of culinary skill. In many cases, they stated that this matter was due to their view of cooking, lack of adequate tutoring by their own mother, occupational constraints, time limitation, and changes that were made in the context of the community due to the prevalence of outdoor and fast foods. The most important categories that emerged regarding the barriers and facilitators of choosing traditional and local foods from the point of women's view will be discussed below.

\section{Cultural context}

Participants expressed that part of the interest and willingness of people to cook is related to the concepts about cookery, their family learning, and parent's perception, which could be effective on their opinions and approach. Also, some people have no cookery skills and experience because their mother did not allow them to cook before they got married:

As long as I was single, I did not cook, because our mother did not agree that her daughter cook foods. Even my mother did not allow us to buy because she did not accept our manner. (a 44-year-old female)

Table 1 Categories and subcategories emerged from data analysis

\begin{tabular}{ll}
\hline Categories & Sub-categories \\
\hline Cultural context & $\begin{array}{l}\text { Family teaching in the field of cooking } \\
\text { Barriers to experience food preparation } \\
\text { during lifespan } \\
\text { Pay attention to the concepts of } \\
\text { traditional medicine } \\
\text { Ethnic pattern in occasions and ceremonies } \\
\text { Customary beliefs about local and } \\
\text { traditional foods }\end{array}$ \\
$\begin{array}{ll}\text { Priority to other life affairs } \\
\text { Interests of family members } \\
\text { Common compliments in the community } \\
\text { Convenience, accessibility, } & \text { Lack of suitable conditions and ingredients } \\
\text { and skills } & \text { Lack of adequate skills in food preparation } \\
\text { Religious considerations } & \text { Halal and haram foods } \\
& \text { Religious rituals }\end{array}$ \\
\hline
\end{tabular}


Participants stated they consumed certain special foods only during special occasions and ceremonies based on the ethnic patterns. A woman pointed out:

Sometimes, in special occasions that my family, such as brothers and sisters join us, there are certain condition that is also defined with some specific foods. (a 62-year-old female).

In this study, some participants mentioned that their food choices were affected by traditional medicine. In this approach, food is not just an edible ingredient eaten to satiate and provide nutrition, but a means to influence the physical and psychological state of the human body, so the precision in choosing food is perceived as important by certain participants for thermoregulation:

My daughter, my sister, and my family, sometimes complain to me about eating fatty foods. I say in our traditional medicine, it is advised eating fatty foods to have a mild temperament, because of the air dryness and coldness. (a 39-year-old female)

Almost all foods prepared and cooked in one region are rooted in the customs of that area. Participants pointed out the local foods are better and healthier than fast food, and they have a great impact on the health and disease of the community.

Sometimes we eat some local food. I taught my children that they eat traditional food too, for example, Kashk contains very calcium, and I think it was more natural and healthier. (39 years old)

Another woman also mentioned:

I think that local food is far healthier. I think when my children eat traditional foods, it's better for mental and physical health. (43 years old).

\section{Social motivations}

Some participants expressed an innate lack of interest in cooking, suggesting that they preferentially address other responsibilities.

I'm not interested in cookery, I do not like it, and I always say that the worst part of house is kitchen, but my mom prepared food with love, I cook food just for eating. (53 years old)

This attitude was observed in most employed women who have less tendency to spend significant time in the kitchen and prefer to do other tasks at home or attending to their own personal work. An educated woman who was employed stated:

Sometimes, I have no patience to cook at all, because I don't like it. I love every work at home, except cooking. I try to use the foods that exist at home. Even if I am forced to eat fried potatoes with eggs (43 years old)

Some participants expressed that their husbands' lack of interest for eating local food has resulted in decreased selection of this type of food. As a young woman said:

I like the local food, it's very good, especially yellow oil, which has a great impact on the taste of foods, but I try to put them aside, because my husband does not like (a 30-year-old female).

The lack of interest among children was the other reasons for avoiding women for preparing and cooking traditional and local food:

I like traditional foods like A'sh, or I like animal oil to use in preparing Halwa (a kind of sweet). I make it and I love it, but my children do not like these foods. (a 34-year-old female)

Some participants did not like choosing these types of foods, and they were forced to consume them under special conditions or during customary occasions, as an elderly woman stated:

I mean, you must inevitably eat something, but I don't have a special local food myself (a 65-year-old female)

Under most circumstances I prefer to eat routine food, but local foods may be something special and you like it in a specific condition. (a 36-year-old female).

\section{Convenience, accessibility, and skills}

Several participants pointed out problems in the preparation of these types of foods that prevented them from choosing traditional foods. Most of these problems included the lack of proper living conditions and the lack of availability of the necessary raw ingredients to prepare them.

If we like eating the local cuisine, it is not in agreement with the tastes of today's children, because we lived and grew up in Tehran. In addition, I cannot cook every local food. I might be able to get the ingredients and cook them, but I'm not sure that it 
gives me a real taste. I'm not sure. (a 44 years-old female)

In previous times, we made "Tarkhineh" (a dry ingredient for cooking A'sh that is obtained by drenching wheat or barley grains in dough and then drying them), but we don't have them now. We eat less of the local foods, but in the Spring we go there and bring this local herb fresh (a 55-year-old female).

Of course, part of this kind of thinking was due to lack of enough skill to cook different foods.

I do not cook A'sh at all, I learned how to prepare it, but I tried to cook it once and unfortunately it was not good, so I did not make it any more (a 43-yearold female).

\section{Religious considerations}

Religious beliefs and customs are one of the other factors which influence our food choices, as stated in the study participants. They spoke about the halal and haram considerations in their food and expressed their perceptions of this religious belief. According to most participants, life in Iran ensured them that it is not necessary to concerned about the choice of haram foods, but this attitude towards imported food was less observed.

I did not pay much attention to this. I always thought that I am in an Islamic country and all these foods are Halal. (a 61-year-old female)

On the other hand, the role of religious occasions in peoples eating patterns is one of the other issues mentioned in the study. Periods such as Ramadan, Muharram, and religious feasts are the times when participants believe changes in their eating patterns are taking place. Usually, at these particular times, some traditional foods may be consumed in certain areas that are very uncommon in other times of the year, such as confectioneries and local foods.

In the month of Ramadan, we consume lots of sweets and soft foods like A'sh, Haleem (a semi-liquid food that prepared with wheat), and rice. (a 46-year-old female)

In the month of Ramadan, our eating habits change slightly. Well, there are certain conditions that must necessarily be taken into consideration for food or a special way of serving our food. We do not have any fried food. We eat a light meal for Iftar (is a meal which Muslims end their daily Ramadan fast at sunset), besides making a Halwa and the other traditional sweets, which is for the month of Ramadan. (a 43-year-old female).

\section{Discussion}

The findings of this study showed that, in a diverse cohort of Iranian women, barriers and facilitators of traditional and local foods choice can include concepts such as "cultural contexts," "social motivations," "convenience, accessibility, and skills," and "religious considerations". The major determinant of food preference is the individual backgrounds that have existed since the beginning of life and affect all aspects of their lives. Such factors may have been unconsciously influencing food choices in many cases and influences one's personality and tendencies, dislikes, and food beliefs.

In this study, we explored broad concepts that are rooted in Iranian culture such as cultural meanings and women's attitudes towards cooking, local food, traditional medicine, and some considerations such as halal food which affect the type, quantity, and manner of eating. Culture is an inclusive and powerful foundation that affects all food choices. People classified the foods as the same ways that exist in their culture, subculture, and ethnic groups to select the food that are favorite acceptable for them. According to participants' statements, their food choices are sometimes affected by a lack of culinary skill. In many cases, they clarified that having no skills was due to their aversion to cooking, lack of adequate education, occupational and time constraints, not matching with children's interest, lack of proper living conditions, poor availability of the necessary specialty ingredients, and increased availability and consumption of fast food, which is in line with the study from Farahmand and colleagues [13].

Many participants indicated that due to the usefulness of traditional foods, they preferred local and traditional food to industrial foods. However, some people were forced to choose processed and semi-prepared foods because of job constraints, citing obstacles in preparing and cooking traditional foods. When modern nutrition transition occurred in traditional society, high-fat and saturated fat-rich foods became dominant, while refined sugar replaced whole grains in the diet. This process is influenced by urbanization and access to processed foods that have high levels of fat, salt, and sugar [14]. In a study conducted by Julie Brimblecombe among Australian indigenous people, subjects choose unhealthy foods unconsciously due to poor understanding of the new food system. Indeed, low awareness of the traditional food system among young people, few facilities for transferring trainings to younger generations, the 
gradual dimming of the role of parents and the elderly, and easy access to new food and the role of the media were the most important factors influencing this type of choice [15]. Similarly to the Australian indigenous, the participants in this study also mentioned the lack of facilities for preparing and less access to the ingredients as the barriers for the low consumption of traditional foods.

Participants in this study expressed their views on halal and haram food, religious beliefs, and the role religious occasions, such as Ramadan and Muharram, in people's eating. These changes mainly involve the increased consumption of specific foods into the diet. People in middle and lower social classes emphasize the consumption of halal food to a greater degree than the high social classes. They considered halal food both religiously and in terms of obtaining a legal income. Mutsikiwa also observed a negative relationship between the social class and the perception about halal food and, in fact, wealth and higher social class did not affect the consumption of halal foods; however, this finding contradicts Max Weber's theory regarding to the effect of social classes on consumption [16].

The greater employment of women represents a shift that complicates this study, as they often lack the time to prepare food at home. In Iran, research on food consumption among women showed that female occupation was the only significant predictor of the consumption of industrial, fast and outdoor foods [17]. Generally, food choices have always been one of the main challenges in dietary intake, especially with the increase in employed women and insufficient time to cook food [18-20]. This barrier is greater in traditional foods than fast foods, due to the time-consuming nature of their preparation, and can play a significant role in selection. In addition, social barriers and other priorities of life have an effective role in driving the selection and preparation of local and traditional foods [21-23].

The food structure and sensory appeals are additional important considerations arising from the study of traditional food systems. The food structure explains seasonal access to food and how to adapt culture to species diversity, and sensory elements include flavors, color, ingredients, texture, and odor of traditional foods [24]. In food choice studies, the physical and sensory properties of food are one of the most important determinants of choice and individual food preferences [19, 20]. Naturally, flavor is considered an indispensable criterion for food choice. Foods that are considered to be hedonically pleasing from the consumer's point of view are likely to be consumed again, for instance, the smell and taste of local foods in each area can be functioned as stimuli for food tourism [25]. As well as these factors mentioned, there appear to be additional determinants such as the motivation for changing the customary diet, having new experiences, age, education level, health concerns, and cost-savings $[6,25,26]$.

Today, there is a growing need for traditional and local food systems as these are key to sustainable nutrition in ecological communities. Nutrition and the methods of food production and consumption, especially indigenous knowledge, can be a potent determinant of sustainable development, as well as the management of emerging non-communicable diseases in contemporary times and ultimately health promotion $[27,28]$.

One of the primary strengths of this study is that we have focused on the perception of women as key members in choosing food, constructing household habits, and shaping food beliefs [29]. In addition, the present study was conducted by purposive sampling with the highest diversity which entails the inclusion of all strata and beliefs in the community, in order to reach a comprehensive perspective. The main weaknesses of the present study lie in the fact that the study was conducted solely in Tehran, which has the least traditional community and food pattern. If this study was conducted in an alternative rural community, then it is likely that the consumption of and attitudes towards local and traditional foods may be ostensibly different.

\section{Conclusion}

The present qualitative study was performed through a phenomenological approach and concluded that, considering the diversity of local and traditional foods in Iranian culture and the positive aspects of these foods, people should be encouraged to consume these foods targeted sustainable nutrition and health in communities. Therefore, emphasizing the special role of women in the household as a member that influences the family food habits, it is necessary to educate them with the aim of reestablishing and promoting the local and traditional food patterns.

\section{Acknowledgements \\ The authors would like to acknowledge and appreciate all participants in this study for their contribution in sharing their views.}

\section{Authors' contributions}

AHR designed and implemented the study and collected and interpreted data. AV supervised the research, JR and AMB helped to write and edited the manuscript. All of the authors approved the final manuscript.

\section{Funding}

This study was funded by National Nutrition and Food Technology Research Institute, Shahid Beheshti University of Medical Sciences to support the implementation of research.

\section{Availability of data and materials} Not applicable

Competing interests

The authors declare that they have no competing interest. 


\section{Author details}

'Department of Community Nutrition, Department of Food and Nutrition Policy and Planning Research, National Nutrition and Food Technology Research Institute, Faculty of Nutrition Sciences and Food Technology, Shahid Beheshti University of Medical Science, No. 7, Hafezi (West Arghavan) St., Farahzadi Blvd., Qods Town, 1981619573P.O. Box: 19395-4741, Tehran, Iran. ${ }^{2}$ Department of Anthropology and Health Studies, Faculty of Social Sciences, Tehran University, Tehran, Iran. ${ }^{3}$ Department of Community Nutrition, National Nutrition and Food Technology Research Institute, Shahid Beheshti University of Medical Science, Tehran, Iran.

\section{Received: 4 September 2019 Accepted: 3 December 2019}

Published online: 30 December 2019

\section{References}

1. Gunkel AH. Food and culture. A Companion to Popular Culture 2016:245-264

2. Jordana J. Traditional foods: challenges facing the European food industry. Food Res Int. 2000;33(3):147-52.

3. Kuhnlein HV, Receveur O. Dietary change and traditional food systems of indigenous peoples. Annu Rev Nutr. 1996;16(1):417-42.

4. Guerrero $L$, et al. Consumer-driven definition of traditional food products and innovation in traditional foods. A qualitative cross-cultural study. Appetite. 2009;52(2):345-54.

5. Mensah P, Tomkins A. Household-level technologies to improve the availability and preparation of adequate and safe complementary foods. Food Nutr Bull. 2003;24(1):104-25.

6. Pieniak $Z$, et al. Association between traditional food consumption and motives for food choice in six European countries. Appetite. 2009;53(1):101-8.

7. Jessri $\mathrm{M}$, et al. Comparison of trends in dietary pattern in Iran, Middle Eastern and North African countries from 1961 to 2005. Pajoohandeh J. 2011:16(1):1-10 Article in Persian.

8. Karizaki VM. Ethnic and traditional Iranian rice-based foods. J Ethn Foods. 2016:3(2):124-34.

9. Creswell JW, Creswell JD. Research design: qualitative, quantitative, and mixed methods approaches. Los Angeles, CA: Sage; 2017

10. Corbin J, Strauss A. Basics of qualitative research. 4th ed. Los Angeles, CA: Sage; 2015.

11. Leech NL, Onwuegbuzie AJ. Qualitative data analysis: a compendium of techniques and a framework for selection for school psychology research and beyond. Sch Psychol Q. 2008:23(4):587.

12. Elo $\mathrm{S}$, et al. Qualitative content analysis: a focus on trustworthiness. SAGE Open. 2014:4(1):1-10. https://doi.org/10.1177/2158244014522633.

13. Farahmand $M$, et al. Barriers to healthy nutrition: perceptions and experiences of Iranian women. BMC Public Health. 2012;12(1):1064.

14. Popkin BM. The nutrition transition and obesity in the developing world. J Nutr. 2001;131(3):871S-3S.

15. Brimblecombe J, et al. Factors influencing food choice in an Australian Aboriginal community. Qual Health Res. 2014;24(3):387-400. https://doi.org/ 10.1177/1049732314521901.

16. Mutsikiwa $\mathrm{M}$, Basera $\mathrm{CH}$. The influence of socio-cultural variables on consumers' perception of halal food products: a case of Masvingo urban, Zimbabwe. Int J Bus Manage. 2012;7(20):112.

17. Beiranvandpour $\mathrm{N}$, et al. Factors affecting the consumption of fast foods among women based on the social cognitive theory. J Educ Community Health. 2014;1(1):19-26 [Article in Persian].

18. Connors $M$, et al. Managing values in personal food systems. Appetite. 2001; 36(3):189-200.

19. Kamphuis CB, de Bekker-Grob EW, van Lenthe FJ. Factors affecting food choices of older adults from high and low socioeconomic groups: a discrete choice experiment. Am J Clin Nutr. 2015;101(4):768-74.

20. Lake $A$, et al. Combining social and nutritional perspectives: from adolescence to adulthood (the ASH30 study). Br Food J. 2009;111(11):1200-11.

21. Blaylock J, et al. Economics, food choices, and nutrition. Food Policy. 1999; 24(2-3):269-86.

22. Jabs J, Devine CM. Time scarcity and food choices: an overview. Appetite. 2006;47(2):196-204.
23. Jabs J, et al. Trying to find the quickest way: employed mothers' constructions of time for food. J Nutr Educ Behav. 2007;39(1):18-25.

24. Vedadhir A, et al. Local - traditional food system: an anthropological research among lak people in rural and nomadic areas of lorestan province. J Rural Dev. 2014;6(1):71-106 [Article in Persian].

25. Kim YG, Eves A, Scarles C. Building a model of local food consumption on trips and holidays: a grounded theory approach. Int J Hosp Manag. 2009;28(3):423-31.

26. Wang $\mathrm{O}$, et al. Motives for consumer choice of traditional food and European food in mainland China. Appetite. 2015;87:143-51.

27. Claxton M. Indigenous knowledge and sustainable development. In 3rd Dation Distingushed Lecture. The cropper found UWI, St Tobago Gustine, Trinidad, 2010

28. Gupta AD. Does indigenous knowledge have anything to deal with sustainable development. Antrocom Online J Anthropol. 2011;7(1):57-64.

29. Farahmand $M$, et al. What are the main barriers to healthy eating among families? A qualitative exploration of perceptions and experiences of Tehranian men. Appetite. 2015;89:291-7.

\section{Publisher's Note}

Springer Nature remains neutral with regard to jurisdictional claims in published maps and institutional affiliations.
Ready to submit your research? Choose BMC and benefit from:

- fast, convenient online submission

- thorough peer review by experienced researchers in your field

- rapid publication on acceptance

- support for research data, including large and complex data types

- gold Open Access which fosters wider collaboration and increased citations

- maximum visibility for your research: over $100 \mathrm{M}$ website views per year

At BMC, research is always in progress.

Learn more biomedcentral.com/submissions 